\title{
Osteoarthritis, Corticosteroids and Role of CYP Genes in COVID-19 Patients: A Mini Review
}

\author{
Usha Adiga $^{1}$ (D), Sachidananda Adiga² ${ }^{(D)}$, Varashree Bolar Suryakanth ${ }^{3 *}$ (D) \\ and Desy Thayyil Menambath ${ }^{1}$
}

\begin{abstract}
${ }^{1}$ Department of Biochemistry, KS Hegde Medical Academy, Nitte (Deemed to be University), Mangalore - 575 018, Karnataka, India.

2Department of Pharmacology, KS Hegde Medical Academy, Nitte (Deemed to be University), Mangalore - 575 018, Karnataka, India.

${ }^{3}$ Department of Biochemistry, Kasturba Medical College, Manipal, Manipal Academy of Higher Education, Manipal - 576 104, Karnataka, India.
\end{abstract}

\begin{abstract}
Objectives of this review is to evaluate the role of cytochrome P450 gene polymorphisms in COVID-19 infected patients with pre-existing OA on corticosteroids. The purpose of this review is to analyze whether polymorphisms of Cytochrome p450 isoforms (CYP2C9 and CYP3A4) affect the dosage of steroids in OA patients in COVID-19 infected patients. This review may provide more therapeutic options; suggest a few guidelines which may be useful in managing COVID-19 patients with pre-existing osteoarthritis. The important role of corticosteroids in treating patients infected with COVID-19 with preexisting osteoarthritis, its influence on incidence of mortality or morbidity may be highlighted. The influence of CYP enzymes and their polymorphisms suggest safety of treatments as well as the possible need for the dosage adjustment or their discontinuation.
\end{abstract}

Keywords: COVID-19, Osteoarthritis, Corticosteroids, Cytochrome P450, Polymorphism

\footnotetext{
*Correspondence: Email: varashree.bs@manipal.edu

(Received: June 27, 2021; accepted: September 28, 2021)

Citation: Adiga U, Adiga S, Suryakanth VB, Menambath DT. Osteoarthritis, Corticosteroids and Role of CYP Genes in COVID-19 Patients: A Mini Review. J Pure App/ Microbiol. 2022;16(1):18-25. doi: 10.22207/JPAM.16.1.28

(C) The Author(s) 2022. Open Access. This article is distributed under the terms of the Creative Commons Attribution 4.0 International License which permits unrestricted use, sharing, distribution, and reproduction in any medium, provided you give appropriate credit to the original author(s) and the source, provide a link to the Creative Commons license, and indicate if changes were made.
} 


\section{INTRODUCTION}

Osteoarthritis (OA) is one of the most common joint disorders which affect around $80 \%$ of individuals above 55 years of age. Age is an independent risk factor for the severe COVID-19 infection. OA patients often exhibit a large spectrum of coexisting co-morbid conditions like diabetes mellitus, cardiovascular disorders and inflammation, which may increase complications of COVID-19 patients. Various drugs used in treating COVID-19 infection, corticosteroids, opioids, NSAIDs, paracetamol etc. have a wide range of iatrogenic influence, which potentially increase the incidence of COVID-19 secondary infection or complications. In this study, the role of corticosteroids and the influence of genes that metabolize these drugs, used to manage OA on the course of COVID-19 infection will be reviewed. As the pandemic with COVID-19 infection continues over a period of time, the conservative management of osteoarthritis may need an adjustment so as to avoid exposure of the patients to added risk factors. The main goal of this review is to analyze whether polymorphisms of Cytochrome p450 isoforms affect the dosage of steroids in OA patients with COVID-19. It may provide more treatment options, with added guidance to the treatment protocol of OA during the pandemic of COVID-19. The susceptibility to the complications of COVID-19 which are life threatening and the greater incidence of morbidity or mortality due to the infection with COVID-19 may act as a guide to orthopaedic surgeons regarding the safe treatment modalities and also regarding the possible need for the dosage adjustment or discontinuation.

Pharmacogenetics is the branch of science that explains the influence of genetic variations on the drug metabolism, drug targets or pathways of the disease resulting in varying drug response in terms of its efficacy or adverse drug reaction. The concept of "personalized medicine" is the present trend and a paradigm shift has been observed from the previous concept of "one drug fits all" to "right drug for the right patient at the right dose and time". By combining modern medicine with molecular advances, the personalized medicine (PM) could target patients individually and improve the effectiveness and efficacy of the therapeutic approach. ${ }^{1}$ Personalized medicine is effective because of the difference in the genetic composition of individuals. In the act of medication, PM has worked with hereditary based evaluation of viability, antagonistic drug reactions, and appropriate dose regimen. ${ }^{2}$ PM translated from System Biology clarifies the mechanism based illness improvement, infection hazard assessment, preventive medication, personalized sickness, and therapy observing. ${ }^{3}$ The development of methods to detect minute changes in molecular profiles in the early stages of disease development is where systems biology is most beneficial in preventive PM. In terms of Systems Pharmacology, integration into PM aims to identify the genes and proteins involved in drug treatment and resistance, so that they can be targeted to improve treatment outcomes through a synergistic impact. ${ }^{4}$ Currently various drugs are used in the treatment of COVID-19, without well established efficacy or safety. The use of pharmacogenomics is worth noting potential limitations in the treatment of COVID-19. ${ }^{5}$

It is very essential to investigate the possible roles of gene polymorphisms that affect the pharmacokinetics and pharmacodynamics of steroids so as to get to know inter-individual variations.

\section{Corticosteroids (CS), OA and COVID-19}

Corticosteroids are potent antiinflammatory drugs used in treating osteoarthritis. ${ }^{6}$ These drugs are administered systemically as well as intra-articularly in OA patients. Prednisolone is the most commonly used steroid administered systemically among others. ${ }^{7} \mathrm{~A}$ few other molecules like methylprednisolone, betamethasone triamcinolone, dexamethasone, triamcinolone are administered as intra-articular injections. ${ }^{8}$ Corticosteroids bring about both anti- inflammation and immunosuppressive, with complex mechanism of action.

Corticosteroids' physiological and pharmacological actions are mediated by their binding to the glucocorticoid receptor(GR). ${ }^{9}$ Upon ligand binding, the glucocorticoid receptor translocates to the nucleus of cells, where it binds to $G C$ response elements (GREs) and controls the transcription of thousands of genes. The GREs are subdivided into 3 broad categories - i)simple- GR binds as homodimer, ii)composite - GR binds along with another transcriptional regulators, 
iii)Tethering- GREs recruited through protein: protein interactions [eg:- Activator Protein1 (AP1) or nuclear factor- $\mathrm{KB}$ (NF-KB)]. GR can function as a monomer at composite and tethering GREs. Although GR can stimulate or repress transcription from all three types of GREs, it is thought that GR works as a transcriptional activator at a normal palindromic GRE, whereas recruitment to tethering sites often results in repression. The tethering (also known as transrepression) of AP1 and NF-KB activity, beleived to account for suppression of inflammatory and immunological responses which are GC-mediated. ${ }^{10}$ In fact, corticosteroid exposure alters leukocyte trafficking (into, through, and out of the stromal compartments of the bone marrow, thymus, and peripheral tissues), immune cell activation, proliferation, and apoptosis, as well as the creation and suppression of inflammatory mediators. ${ }^{11}$ The anti-inflammatory and immunosuppressive effects are desirable, but the metabolic and toxic effects are not, as they are the origin of the negative outcomes. ${ }^{11}$ In a positive feedback loop of pathogenic inflammation, if coordinated recruitment of innate and adaptive immunity fails to successfully manage the pathogen, more immune cells will be activated. This results in the release of additional cytokines. This condition is known as cytokine storm syndrome (CSS), and it has been linked to ARDS and multiple organ failure in extreme cases. ${ }^{12}$

In COVID-19 patients the expression of IL-1B, IFN- $\gamma$, IP-10, and monocyte chemoattractant protein 1 (MCP-1) were found to be high. These inflammatory cytokines may activate the T-helper type 1 (Th1) cell response. ${ }^{13}$ The activation of Th1 cells is a crucial step in the activation of specialised immunity. ${ }^{14}$ Elevated levels of Th2 cell-secreted cytokines (such as IL-4 and IL-10), inhibit the inflammatory response were also seen in patients with COVID-19. A positive correlation was seen between the severity of the disease and inflammatory markers like serum IL-2R and IL-6 in patients with COVID-19. ${ }^{15}$ Other studies have found that, compared to COVID-19 patients from general wards, patients in the intensive care unit (ICU) display increased serum levels of granulocyte colony-stimulating factor, IP-10, MCP-1, macrophage inflammatory protein- $1 \mathrm{~A}$, and TNF- $\alpha$. A strong positive correlation was suggested between the cytokine storm and the severity of the disease. ${ }^{13}$ In COVID-19, the inflammatory cytokine storm is closely related to the development and progression of ARDS and multiple organ failure.

Timely administration of glucocorticoids inhibits inflammation in case of cytokine storm in the initial phases. ${ }^{16}$ It also effectively prevents ARDS and safeguards patients' organ functions. For patients who are severely ill, with oxygenation indicators being in declining mode, with a fast imaging progress, and excessive inflammatory response, the short term (3-5 days) use of glucocorticoids may be appropriate. Administration of methylprednisolone at a dose of $1-2 \mathrm{mg} / \mathrm{kg} /$ day would be the most recommended. ${ }^{17}$ However a large dose of steroids may cause immunosuppression and thereby delay the SARS-CoV-2 clearance.

They act by inhibiting inflammatory cells, metalloproteases and metalloprotease activators. They also inhibit the pro-inflammatory factors by inhibiting their synthesis and secretion. ${ }^{18}$ These agents when used on long term basis, orally or parenterally, have been reported to be associated with adverse events like diabetes mellitus, osteoporosis, superinfection, CVD and immunosuppression. ${ }^{19}$ Adverse events are less likely with intra- articular administration, probably because of decreasing cortisol levels in blood within a few hours which returns to the baseline within 1-4 weeks. Intra-articular corticosteroids administration reduces inflammatory markers like CRP and ESR that lasts for a few months. It also results in a rise in plasma glucose in osteoarthritis cases with pre-existing diabetes. ${ }^{20}$

A registered review in Cochrane analyzed published studies that evaluated systemic corticosteroid treatment for community acquired pneumonia, administered along with antibiotics, as compared to placebo or those without corticosteroids. ${ }^{21}$ The combined treatment declined the rates of mortality as well as morbidity in severe pneumonia cases. Decreased morbidity was noted in adults and children, but not mortality with pneumonia. Hyperglycaemia was the adverse effect reported in agreement with an another review that included the clinical trials on pneumonia cases. ${ }^{22}$

The situation regarding viral infections of the respiratory tract and effect of corticosteroids on complications and adverse events is inconclusive. 
Severe acute respiratory syndrome (SARS)-CoV outbreaks of 2002 were widely treated with corticosteroids ${ }^{23}$ and Middle East respiratory syndrome (MERS)-CoV of 2012 as well. ${ }^{24}$ A randomized control trial reported that, the SARS-CoV RNA in plasma in cases on ribavirin who were treated with hydrocortisone therapy was higher compared to placebo. ${ }^{25}$ Corticosteroid treatment leads to a delay in MERS coronavirus RNA clearance from the respiratory tract secretions as reported in MERS study. ${ }^{24} \mathrm{~A}$ meta-analysis covering a large population of influenza pneumonia patients, corticosteroids were reported to be associated with higher mortality and a greater rate of secondary infection. ${ }^{26}$ Patients with RSV infection, on steroid therapy were reported to have an impaired antibody response; however no significant differences were noted in viral load peak. ${ }^{27}$ Contradictory reports are also available that support the utility of corticosteroids at a low to moderate dosage in SARS infected cases. Corticosteroids were reported to contribute to lower mortality and shorten hospital stay, in a retrospective study. ${ }^{28}$ Pneumonia due to influenza a infection, corticosteroid administration in low to moderate dose were reported to reduce 30 day mortality in a prospective cohort study. ${ }^{29}$ Guidelines from the American Thoracic Society and the Infectious Diseases Society of America advises added corticosteroid therapy only if there are other indications in patients with pneumonia or influenza pneumonia. ${ }^{30}$

Until today, no anti-COVID-19 medicines have been approved by the WHO. Remdesivir, an RNA polymerase inhibitor and experimental $\mathrm{C}$-adenosine nucleoside prodrug, is one of the few medications used to treat COVID-19 that has had some success. ${ }^{31}$ It has been established in multiple clinical trials to be a relatively safe medicine with linear pharmacokinetics. ${ }^{31}$ Another RNA polymerase inhibitor that has been tested on COVID-19 patients is favipiravir. Due to the non-CYP metabolic route of favipiravir, pathophysiological variables in COVID-19 patients are unlikely to have a major impact on favipiravir disposition. ${ }^{32}$

The combination of lopinavir and ritonavir, which was licenced for the treatment of HIV, has been tested for protease inhibition against other coronaviruses, including SARS-CoV-2 in vitro and in COVID-19 patients. Some studies have found efficacy (e.g., length of ICU stay, viral load clearance), whereas others have found no difference in COVID patients when compared to the comparator. ${ }^{33}$

Chloroquine reduces the generation and release of TNF and IL- 6 , suggesting that it may reduce the cytokine storm in COVID-19 infected patients. ${ }^{34}$ Another repurposed medicine for COVID-19, hydroxychloroquine, has been found to be less hazardous than chloroquine for inhibiting SARS-CoV-2 infection in vitro. By limiting T-cell stimulation and thus decreasing cytokine production, hydroxychloroquine can diminish the inflammatory response and cytokine storm (aggressive inflammatory response with the release of a significant amount of proinflammatory cytokines) in individuals severely infected with SARS-CoV-2. It can also prevent pro-inflammatory genes from being expressed by disrupting the interaction between DNA/RNA and toll-like receptors. ${ }^{35,36}$

According to the initial report on COVID-19 infected patients receiving corticosteroids reported no association between the therapy and time taken for clearing viremia, hospital length of stay, or duration of symptoms. ${ }^{37}$ However no clinical data is available that suggest the overall beneficial or detrimental role of corticosteroids in COVID-19 patients. Some light on future research prospects and indications for the use of corticosteroids for the treatment of COVID-19 has to thrown. Indication of the drug might be due to its immunosuppressive and anti inflammatory actions. There are no clear indications, however WHO guidelines suggests to avoid routine corticosteroid administration unless the usage is indicated in COVID-19 infection. ${ }^{38,39}$ Patients with osteoarthritis already treated with systemic corticosteroids, there is a lack of guidelines that suggest the discontinuation of steroids in COVID 19 patients. ${ }^{40}$

\section{Possible Role of involvement of CYP2C9 and CYP3A4 Genes in drug metabolism}

The Cytochrome $\mathrm{P} 450$ system consists of a set of enzymes involved in drug metabolism having an active role in the phase I reactions. Enzymes of cytochrome p450 are mainly expressed in the hepatic tissue; however, they are also expressed in other tissues, like kidneys, skin, intestine, lung, and brain. ${ }^{41}$ The enzyme CYP3A4 is responsible for the metabolism of $50 \%$ of medications. The remaining 
$35-40 \%$ of the metabolism occurs through other variants of cytochrome P450.

CYP3A4 is a constitutively expressed variant of CYP system expressed in hepatic tissue. ${ }^{42}$ Substrates for the cytochrome P450 enzymes can be either endogenous like lipid derivatives and retinoids or exogenous like chemicals, drugs or plant products. The members of cytochrome p450 metabolize more than $80 \%$ of the commonly prescribed medications.

Enzymes of cytochrome p450 that are expressed in hepatic tissues are found to be polymorphic. Commonly observed polymorphisms were that of CYP2C9, CYP2C19, and CYP2D6 ${ }^{43} \mathrm{CYP}$ gene mutations lead to under expressed, abolished or over expressed CYP protein products which leads to altered drug metabolism by a specific enzyme. This may lead to a potential adverse drug reaction in the host. Adverse reactions of drugs are a great burden, estimated cost being more than $\$ 100$ billion annually and it may be responsible for around 100,000 deaths per year in USA. ${ }^{43}$

Health data available from COVID-19 patients have suggested an elevation of liver transaminases ( AST, ALT). The data also suggests a severe hepatic stress during COVID-19 infection. ${ }^{44-46}$ Elevated cytokine levels suppress the expression of CYP, thereby interfering with the drug metabolisms, resulting in decreased drug elimination. Drug toxicity effects can be observed locally as well as systemically within $48-72$ hours of active viral infection. CYP enzymes like CYP3A4, CYP2B6, and CYP2C9 are downregulated during cytokine storm of COVID-19.Cytokines are responsible for the altered hepatic CYP expression. ${ }^{47}$ Regulation of CYP enzymes may vary, either a single cytokine may influence the expression of multiple enzymes or multiple cytokines may alter the single enzyme expression.

Corticosteroids are another group of medications that have emerged as a treatment option for COVID-19. The association of glucocorticoids (GC) and the enzymes of Cytochrome P450 family is complex. GCs are metabolized partially by CYP3A enzymes. Hence co-administration of CYP inhibitors with steroids causes over suppression of hypothalamus pituitary adrenal axis and enhances the effects of exogenous GCs. ${ }^{48}$ Contrary to these, GCs also induce the enzymes of cytochrome P450. In vitro experiments on mammalian cell lines are available that evaluated the association between GCs and CYP and in vivo studies performed in rodents, focused on CYP2C11. ${ }^{49} \mathrm{~A}$ significant risk reduction was observed in patients requiring oxygen supplementation or those on ventilators with the administration of steroids compared to usual care group in the RECOVERY trial. ${ }^{50}$ Dexamethasone acts both as an inducer as well as a substrate for the CYP3A4 enzyme. ${ }^{51,52}$ Hyperglycemia and fluid retention may be the major adverse effects of reduced dexamethasone metabolism. ${ }^{53}$

\section{Future Research Perspectives}

A study may be designed that evaluates the pattern of CYP3A4 and CYP2C9 polymorphism in patients with COVID-19 infection with preexisting OA on corticosteroids .

Drug effectiveness and toxicity are mainly determined by the genetic background of the individuals. ${ }^{54}$ Studying the genetic composition as well as polymorphisms/mutations plays a crucial role as these can influence the expression patterns of CYP enzymes which in turn influence the drug responsiveness. This emphasizes the role of personalized medicine and opens up a new era in its practice. If there is an association between clinical effectiveness of corticosteroids in reducing inflammation and hence the pain management of OA patients diagnosed as COVID-19, with respect to CYP3A4 and CYP2C9 polymorphism patterns., it may be of great value in personalizing the treatment.

Significance of such study and Impact on society

The study may be able to find the association between mutations of CYP gene polymorphisms on steroid metabolism and its influence on the perception of pain in COVID-19 patients already on steroids with pre-existing $\mathrm{OA}$. This may emphasise the role of CYP gene polymorphism in predisposing OA patients to an added risk of COVID-19. The study may acknowledge the important public health message of careful monitoring of OA patients on steroids with COVID-19. The study emphasises on the pain management by focusing on the polymorphisms of cytochrome p 450 at the genetic level and their association with the steroid metabolism. Anti-inflammatory role of corticosteroids and effectiveness in pain management is determined by the polymorphisms of CYP450. The question 
whether these polymorphisms and their association with steroid metabolism, predispose OA patients to COVID-19 infection will be explored. Additionally, because of the paucity of data and lack of well-designed studies that answer the questions on the efficacy, safety and role of corticosteroids in $\mathrm{OA}$ and its association with COVID-19, our study may provide a permanent solution by providing treatment option to COVID-19 patients with OA at genetic level.

\section{ACKNOWLEDGMENTS}

None.

\section{CONFLICT OF INTEREST}

The authors declare that there is no conflict of interest.

\section{AUTHORS' CONTRIBUTION}

UA drafted the manuscript, compiled information from the literature. VBS carried out literature search. SA supervised and reviewed the manuscript.

\section{FUNDING}

None.

\section{DATA AVAILABILITY}

The datasets generated during and/or analysed during the current study are available from the corresponding author on reasonable request.

\section{ETHICS STATEMENT}

Not applicable.

\section{REFERENCES}

1. Mini E, Nobili S. Pharmacogenetics: implementing personalized medicine. Clin Cases Miner Bone Metab. 2009;6(1):17-24. PMID: 22461093

2. Chen R, Snyder M. Systems biology: personalized medicine for the future? Curr Opin Pharmacol. 2012;2(5):623-628. doi: 10.1016/j.coph.2012.07.011

3. Agyeman AA, Ofori-Asenso R. Perspective: Does personalized medicine hold the future for medicine?. J Pharm Bioallied Sci. 2015;7(3):239-244. doi: 10.4103/0975-7406.160040

4. Wist AD, Berger SI, lyengar R. Systems pharmacology and genome medicine: a future perspective. Genome Med. 2009; 1(1):11. doi: 10.1186/gm11

5. Fricke-Galindo I, Falfan-Valencia R. Pharmacogenetics Approach for the Improvement of COVID-19 Treatment. Viruses. 2021;13(3):413. doi: 10.3390/v13030413
6. Becker DE. Basic and Clinical Pharmacology of Glucocorticosteroids. Anesthesia Progress 2013;60(1):25-32. doi: 10.2344/0003-3006-60.1.25

7. Slomski A. Six Weeks of Prednisolone Reduced Hand Osteoarthritis Pain. JAMA. 2020;323(4):301. doi: 10.1001/jama.2019.20918

8. Ayhan E, Kesmezacar H, Akgun I. Intraarticular injections (corticosteroid, hyaluronic acid, platelet rich plasma) for the knee osteoarthritis. World J Orthop. 2014;5(3):351-361. doi: 10.5312/wjo.v5.i3.351

9. Evans RM. The steroid and thyroid hormone receptor superfamily. Science. 1988;240(4854):889-895. doi: 10.1126/science.3283939

10. Rogatsky I, Ivashkiv LB. Glucocorticoid modulation of cytokine signaling. Tissue Antigens. 2006;68(1):1-12. doi: 10.1111/j.1399-0039.2006.00599.x

11. Coutinho $A E$, Chapman KE. The anti-inflammatory and immunosuppressive effects of glucocorticoids, recent developments and mechanistic insights. $\mathrm{Mol}$ Cell Endocrinol. 2011;335(1):2-13. doi: 10.1016/j. mce.2010.04.005

12. Mehta P, McAuley DF, Brown M, Sanchez E, Tattersall RS, Manson JJ. COVID-19: consider cytokine storm syndromes and immunosuppression. Lancet. 2020;395(10229):1033-1034. doi: 10.1016/S01406736(20)30628-0

13. Huang $C$, Wang $\mathrm{Y}, \mathrm{Li} X$, et al. Clinical features of patients infected with 2019 novel coronavirus in Wuhan, China. Lancet. 2020;395(10223):497-506. doi: 10.1016/ S0140-6736(20)30183-5

14. Marchingo JM, Sinclair LV, Howden AJM, Cantrell DA. Quantitative analysis of how Myc controls T cell proteomes and metabolic pathways during $T$ cell activation. ELife. 2020;9:e53725. doi: 10.7554/ eLife. 53725

15. Chen L, Liu H-G, Liu W, et al. Analysis of clinical features of 29 patients with 2019 novel coronavirus pneumonia. Chin J Tuberc Respir Dis. 2020;43. doi: 10.3760/cma.j.issn.1001-0939.2020.0005

16. Ye Q, Wang B, Mao J. The pathogenesis and treatment of the 'Cytokine Storm' in COVID-19. J Infect. 2020;80(6):607-613. doi: 10.1016/j.jinf.2020.03.037

17. Yuan-Yuan $Q$, Yi-Hong Z, Yan-Qiu L, et al. Effectiveness of glucocorticoid therapy in patients with severe novel coronavirus diseases 2019: protocol of a randomized controlled trial. Chin Med J. 2020;133(9):1080-1086. doi: 10.1097/CM9.0000000000000791

18. Ostergaard M, Halberg P. Intra-Articular Corticosteroids in Arthritic Disease: A Guide to Treatment. BioDrugs 1998;9(2):95-103. doi: 10.2165/00063030-19980902000002

19. Liu D, Ahmet A, Ward LM, et al. A practical guide to the monitoring and management of the complications of systemic corticosteroid therapy. Allergy Asthma Clin Immunol. 2013;9(1):30. doi: 10.1186/1710-1492-9-30

20. Habib GS. Systemic effects of intra-articular corticosteroids. Clin Rheumatol. 2009;28(7):749- 756. doi: 10.1007/s10067-009-1135-x

21. Stern A, Skalsky K, Avni T, Carrara E, Leibovici L, Paul M. Corticosteroids for pneumonia. Cochrane Database Sys Rev. 2017;12(12):CD007720. doi: 10.1002/14651858. CD007720.pub3 
22. Corticosteroids in Community-Acquired Pneumonia. The Journal of the American Medical Association. 2020;323(9):887-888. doi: 10.1001/jama.2020.0216

23. Stockman LJ, Bellamy R, Garner P. SARS: Systematic Review of Treatment Effects. PLoSMed. 2006;3(9):e343. doi: 10.1371/journal.pmed.0030343

24. Arabi YM, Mandourah Y, Al-Hameed F, et al. Corticosteroid Therapy for Critically III Patients with Middle East Respiratory Syndrome. Am J Respir Crit Care Med. 2018;197(6):757-767. doi: 10.1164/ rccm.201706-11720C

25. Lee N, Chan KCA, Hue DS, et al. Effects of early corticosteroid treatment on plasma SARS-associated Coronavirus RNA concentrations in adult patients. J Clin Virol. 2004;31(4):304-309. doi: 10.1016/j. jcv.2004.07.006

26. Ni Y-N, Chen G, Sun J, Liang B-M, Liang Z-A. The effect of corticosteroids on mortality of patients with influenza pneumonia: a systematic review and meta-analysis. Crit Care. 2019;23(1):99. doi: 10.1186/s13054-0192395-8

27. Lee FE-H, Walsh EE, Falsey AR. The Effect of Steroid Use in Hospitalized Adults With Respiratory Syncytial Virus-Related Illness. Chest. 2011;140(5):1155-1161. doi: 10.1378/chest.11-0047

28. Chen R-C, Tang X-P, Tan S-Y, et al. Treatment of Severe Acute Respiratory Syndrome With Glucosteroids. Chest. 2006;129(6):1441-1452. doi: 10.1378/ chest.129.6.1441

29. Li H, Yang S-G, Gu L, et al. Effect of low-to-moderatedose corticosteroids on mortality of hospitalized adolescents and adults with influenza A(H1N1)pdm09 viral pneumonia. Influenza Other Respi Viruses. 2017;11(4):345-354. doi: 10.1111/irv.12456

30. Metlay JP, Waterer GW, Long AC, et al. Diagnosis and Treatment of Adults with Community-acquired Pneumonia. An Official Clinical Practice Guideline of the American Thoracic Society and Infectious Diseases Society of America. Am J Respir Crit Care Med. 2019;200(7):e45-e67. doi: 10.1164/rccm.2019081581ST

31. Doggrell SA. Remdesivir, a remedy or a ripple in severe COVID-19? Expert Opin Investig Drugs. 2020;29(11):1195-1198. doi: $10.1080 / 13543784.2020 .1821645$

32. Dhama K, Sharun K, Tiwari R, et al. CovID-19, an emerging coronavirus infection: advances and prospects in designing and developing vaccines, immunotherapeutics, and therapeutics. Hum Vaccin Immunother. 2020;16(6):1232-1238. doi: 10.1080/21645515.2020.1735227

33. Cao B, Wang $Y$, Wen D, et al. A trial of lopinavirritonavir in adults hospitalized with severe Covid-19. N Engl J Med. 2020;382(19):1787-1799. doi: 10.1056/ NEJMoa2001282

34. Gao J, Tian Z, Yang X. Breakthrough: Chloroquine phosphate has shown apparent efficacy in treatment of COVID-19 associated pneumonia in clinical studies. Biosci Trends. 2020;14(1):72-73. PubMed PMID: 32074550. doi: 10.5582/bst.2020.01047

35. Liu J, Cao R, Xu M, et al. Hydroxychloroquine, a less toxic derivative of chloroquine, is effective in inhibiting
SARS-CoV-2 infection in vitro. Cell Discov. 2020;6:16. doi: 10.1038/s41421-020-0156-0

36. Zhou D, Dai SM, Tong Q. COVID-19: a recommendation to examine the effect of hydroxychloroquine in preventing infection and progression. $J$ Antimicrob Chemother. 2020;75(7):1667-1670. doi: 10.1093/jac/ dkaa114

37. Zha L, Li S, Pan L, et al. Corticosteroid treatment of patients with coronavirus disease 2019 (COVID-19). Med J Aust. 2020;212(19):416-420. doi: 10.5694/ mja2.50577

38. Corticosteroids for covid-19, living guidance. 2020. https://www.who.int/publications/i/item/WHO-2019nCoV-Corticosteroids-2020.1

39. Clinical management of severe acute respiratory infection (SARI) when COVID-19 disease is suspected, 2020. https://www.who.int/docs/default-source/ coronaviruse/clinical-management-of-novel-cov.pdf

40. Hermann W, Lambova S, Muller-Ladner U. Current Treatment Options for Osteoarthritis. Current Rheumatology Reviews. 2018;14(2):108-116. doi: 10.2174/1573397113666170829155149

41. Krishna DR, Klotz U. Extrahepatic Metabolism of Drugs in Humans. Clin Pharmacokinet. 1994;26(2):144- 160. doi: 10.2165/00003088-199426020-00007

42. Shimada T, Yamazaki H, Mimura M, Inui Y, Guengerich FP. Interindividual variations in human liver cytochrome P-450 enzymes involved in the oxidation of drugs, carcinogens and toxic chemicals: studies with liver microsomes of 30 Japanese and 30 Caucasians. J Pharmacol Exp Ther 1994;270(1):414-423.

43. Katzung BG.Basic and clinical Pharmacology - Chapters 3 and 4, Appleton-Lange/McGraw-Hill Publishers, New York. 2014.

44. Li J, Fan JG. Characteristics and mechanism of liver injury in 2019 coronavirus disease. J Clin Transl Hepatol. 2020;8(1):13-17. doi: 10.14218/JCTH.2020.00019

45. Hong KS, Lee KH, Chung JH, et al. Clinical features and outcomes of 98 patients hospitalized with SARS-CoV-2 infection in Daegu, South Korea: a brief descriptive study. Yonsei Med J. 2020;61(5):431-437. doi: 10.3349/ ymj.2020.61.5.431

46. Cai $Q$, Huang $D, Y u H$, et al. COVID-19: abnormal liver function tests. J Hepatol. 2020;73(3):566-574. doi: 10.1016/j.jhep.2020.04.006

47. Zhang J-M, An J. Cytokines, inflammation, and pain. Int Anesthesiol Clin. 2007;45(2):27-37. doi: 10.1097/ AlA.0b013e318034194e

48. Morgan ET, MacGeoch C, Gustafsson JA. Hormonal and Developmental Regulation of Expression of the Hepatic Microsomal Steroid 16aHydroxylase Cytochrome P-450 Apoprotein in the Rat*. J Biol Chem 1985;260(22):11895-11898. doi: 10.1016/S00219258(17)38963-9

49. Pichard L, Fabre I, Fabre G, et al. Cyclosporin A drug interactions. Screening for inducers and inhibitors of cytochrome P-450 (cyclosporin A oxidase) in primary cultures of human hepatocytes and in liver microsomes. Drug Metab Dispos. 1990;18(5):595-606. PMID: 1981707

50. Horby P, Lim WS, Emberson JR, et al. Dexamethasone in hospitalized patients with Covid-19-preliminary 
report. N Engl J Med. 2020;384(8):693-704.

51. Doi M, Kajikawa N, Aiba T. Effects of dexamethasone to reverse decreased hepatic midazolam metabolism in rats with acute renal failure. Xenobiotica. 2020;50(5):506514. doi: 10.1080/00498254.2019.1655680

52. Deb S, Pandey M, Adomat H, Guns EST. Cytochrome P450 3A-mediated microsomal biotransformation of 1 $\alpha, 25$-dihydroxyvitamin D3 in mouse and human liver: drug-related induction and inhibition of catabolism.
Drug Metab Dispos. 2012;40(5):907-918. doi: 10.1124/ dmd.111.041681

53. Kadmiel M, Cidlowski JA. Glucocorticoid receptor signaling in health and disease. Trends Pharmacol Sci. 2013;34(9):518-530. doi: 10.1016/j.tips.2013.07.003

54. Cascella M, Rajnik M, Aleem A, Dulebohn S, Di Napoli R. Features, evaluation, and treatment of coronavirus (COVID-19). StatPearls. 2021. 